ing the new theories concerning the relation of radiant energy to chemical action.

2. The fact that the velocity constants obtained in solution so closely approximate those obtained in the gas phase by Daniels and Johnston is of peculiar significance since, as far as the writer is aware, this is the first time that the velocity of a monomolecular reaction has been measured under so wide a variation of conditions.

3. The experimental facts show the energy of activation or the critical increment to be practically independent of the temperature.

4. It has been shown that, in accordance with a necessary deduction from the Perrin-Lewis radiation theory, a change in reaction velocity brought about by temperature effects or the influence of solvent diminishes as the magnitude of the velocity constant increases.

5. A comparison has been made between the values of the velocity constant experimentally obtained and those calculated from the critical increment, $E$, by means of the equation recently proposed by Dushman. Although the agreement is not exact, it is far better than can be obtained using the older equations developed by Trautz and Lewis.

b. The nitrogen peroxide formed in the reaction has been shown to exert an auto-catalytic effect on the thermal decomposition of the nitrogen peatoxide.

7. This work is at present being extended in this laboratory to other solvents and to a study of the absorption spectrum of nitrogen peroxide in the various solvents.

Madison, Wisconsin

[Contribution from the Laboratory of Physical Chemistry, Princeton UNIVERSITY]

\title{
THE SIZES OF ATOMS IN CRYSTALS
}

\section{By ROBERT N. PEASE ${ }^{1}$}

Received February 1, 1922

The author has recently shown ${ }^{2}$ that molecular volumes, as revealed by the constant $b$ of van der Waals' equation, are consistent with the view that the volumes of atoms and groups of atoms depend upon the number and arrangements of electrons about the positive nuclei of the atoms rather than on the particular kinds of atoms involved. It was shown, for example, that the volumes of the nuclear carbon, nitrogen and oxygen atoms in methane, ammonia and water respectively, are nearly the same and equal to that of the rare gas, neon. The number and arrangements of electrons about the positive nuclei of these nuclear atoms and of neon are the same for all. Similarly, the volumes of the

1 National Research Fellow in Chemistry.

2 Pease, This Journal, 43, 991 (1921). 
oxygen molecule and the carbon atoms in ethylene were shown to be the same. In the present paper, it is proposed to apply this point of view to an examination of the interatomic distances ${ }^{3}$ in crystals of the diamond type.

It is now known that diamond, ${ }^{4}$ silicon, ${ }^{5}$ gray tin $^{6}{ }^{6}$ carborundum, ${ }^{7}$ zinc sulfide, ${ }^{4}$ the cuprous halides, ${ }^{8}$ and silver iodide, ${ }^{9}$ all crystallize in the so-called diamond-type lattice. Neutral carbon, silicon and tin atoms all require 4 electrons to complete their outer shells. It is evident that in the diamond type of lattice the atoms can all acquire these by sharing: pairs of electrons with the 4 surrounding atoms. It is obvious that in the case of carborundum ( $\mathrm{SiC}$ ) the same is true, for a crystal of this substance differs from one of the diamond or silicon only in that half of the atomic kernels are replaced by those of the other element.

For the salts which crystallize in the diamond lattice relations of a similar sort hold. Let it be assumed that in zinc sulfide each zinc atom has lost 2 electrons to a sulfur atom, thereby completing the latter's octet. The zinc atom will then require 8 additional electrons in its outer shell (corresponding to krypton) to complete the latter. Evidently, if it shares with each of the 4 surrounding sulfide ions a pait of electrons belonging to the latter, it can acquire the 8 it needs. There thus appears to be a very definite chemical reason for zinc sulfide crystallizing in the diamond type of lattice. In the cuprous halides and silver iodide the same relations hold. The metal atom may be regarded as giving up 1 electron to the halogen atom, thereby completing the latter's octet and then obtaining the 8 electrons which it requires to fill out its outer shell by sharing electrons with the 4 halide ions surrounding it.

Of the kinds of atoms contained in these substances which crystallize in the diamond lattice, carbon is of the neon type; silicon, sulfur and chlorine are of the argon type; copper, zinc and bromine, of the krypton type; and silver, tin and iodine, of the xenon type. It might be surmised from the conclusions reached in the earlier paper that with a single value for the radius of each of these characteristic types, the inter-atomic distances for all these substances could be derived. This is in fact the case. For the neon type, the value for the radius of the carbon atom in diamond,

${ }^{3}$ This term applies, of course, to the distances between the centers of the atoms.

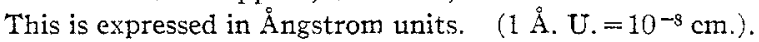
1918.

${ }^{4}$ W. H. and W. I. Bragg, "X-Rays and Crystal Structure." G. Bell and Sons,

${ }^{5}$ Debye and Scherrer, Physik. Z., 17, 277 (1916).

${ }^{5}$ Bijl and Kolkmeijer, Verslag Akad. Wetenschappen Amsterdam, 27, 359 (1918).

${ }^{7}$ Burdick and Owen, This Journal, 40, 1749 (1918). See also Hull, Phys. Rev.,

[2] 13, 292 (1919).

${ }^{8}$ Wyckoff and Posnjak, Thrs Journal, 44, 30 (1922).

9 Wilsey, Phil. Mag., 42, 262 (1921). 
i. e., one-half the distance between nearest atomic centers, $0.77 \AA$. U., is taken. For the argon type, the value for silicon is taken. Debye and Scherrer give this as $\frac{2.35}{2}=1.18 \AA$. U. According to Gmelin-Kraut's Handbuch, ${ }^{10}$ however, numerous determinations place the density of silicon at 2.49 , which gives $\frac{2.30}{2}=1.15 \AA$. U., for the radius of the silicon atom. This is the value used in the calculations. For the krypton type there is available the value for the distance between centers in cuprous bromide, $2.52 \AA$. U. One half this, $1.26 \AA$. U., is taken as the value for the radius of atoms of the krypton type. For the xenon type, the value for gray tin, $\frac{2.80}{2}=1.40 \AA$. U., has been chosen.

Using these four constants, the interatomic distances for the 9 substances have been calculated with the following results.

TABLE I

INTER-ATOMIC DISTANCES

\begin{tabular}{|c|c|c|c|c|c|c|c|c|}
\hline \multirow[t]{2}{*}{ 'Type } & \multicolumn{3}{|c|}{ Radius } & \multicolumn{5}{|c|}{ Distance between nearest centers } \\
\hline & & A. U. & & Calc. & Observed & Diff. & $\begin{array}{c}\text { From Bragg' } \\
\text { radii }\end{array}$ & 's \\
\hline Neon & (C) & 0.77 & Diamond & $1.54^{a}$ & 1.54 & $\cdots$ & 1.54 & \\
\hline Argon & $(\mathrm{Si}, \mathrm{S}, \mathrm{Cl})$ & 1.15 & Silicon & $2.30^{\circ}$ & 2.30 & & 2.35 & \\
\hline Krypton & $(\mathrm{Cu}, \mathrm{Zn}, \mathrm{Br})$ & 1.26 & Gray tin & $2.80^{\circ}$ & 2.80 & & 2.80 & \\
\hline Xenon & $(\mathrm{Ag}, \mathrm{Sn}, \mathrm{I})$ & 1.40 & $\mathrm{SiC}$ & 1.92 & 1.90 & +0.02 & 1.95 & +0.05 \\
\hline & & & $\mathrm{ZnS}$ & 2.41 & 2.35 & +0.06 & 2.35 & 0 \\
\hline & & & $\mathrm{CuCl}$ & 2.41 & 2.38 & +0.03 & 2.43 & +0.05 \\
\hline & & & $\mathrm{CuBr}$ & $2.52^{a}$ & 2.52 & $\ldots$ & 2.57 & +0.05 \\
\hline & & & $\mathrm{CuI}$ & 2.66 & 2.63 & +0.03 & 2.78 & +0.15 \\
\hline & & & $\operatorname{AgI}$ & 2.80 & 2.83 & -0.03 & 3.18 & +0.35 \\
\hline
\end{tabular}

a These values taken as standards.

The agreement is evidently very good. Substances which according to the hypothesis should be the same are gray tin and silver iodide, the values for which are 2.80 and $2.83 \AA$. U., respectively, and zinc sulfide and cuprous chloride, the values for which are 2.35 and $2.38 \AA$. U., respectively. Since the ions of zinc sulfide are doubly charged while those of cuprous chloride are singly charged, one might expect that the interatomic distance would be less in the former. The relatively slight difference between the two $(0.03 \AA$. U.) as compared with that between an alkaline earth sulfide and the corresponding alkali chloride (e.g., $d_{\mathrm{KC} 1}-$ $d_{\mathrm{CaS}}=0.36 \AA$. U.) is good evidence for the close-packed nature of the diamond lattices in which zinc sulfide and cuprous chloride crystallize.

Zinc oxide, though not crystallizing in the diamond lattice, nevertheless has the same relative arrangement of atoms. ${ }^{11}$ The inter-atomic distance is $1.97 \AA$. U. The calculated value would be $0.77+1.25=2.02 \AA$. U.

${ }^{10}$ Gmelin-Kraut, "Handbuch der anorganischen Chemie," Carl Winter, 1912, 3, 1,110 .

${ }^{11}$ W. L. Bragg, Phil. Mag., [6] 39, 647 (1920). 
It is of interest to note that crystals of silver iodide and of gray tin are isosteric, as Langmuir defines the term, in that they differ only in the distribution of net charge among the positive nuclei, the charge on each tin nucleus being 50 units while the charges on the silver and iodine nuclei are 47 and 53 units respectively. The number and arrangement of electrons are the same in both. Zinc sulfide and cuprous chloride crystals are also isosteric.

The manner of regarding inter-atomic distances as depending on the rare gas type of atom involved and on the type of lattice, as exemplified in the above analysis, is suggested as an alternative to W. L. Bragg's method of treating these values. ${ }^{12}$ Bragg considers that the atoms in a crystal can in all cases be regarded as close-packed spheres and that it is possible "to assign to the sphere representing an atom of any element a constant diameter characteristic of that element." As Bragg has pointed out, the resulting atomic radii are not wholly consistent with the present day ideas concerning atoms. He is constrained to assume, for example, that the radius of the sodium ion is $1.78 \AA$. U. while that of the fluoride ion, which has the same rare-gas (neon) structure, is only $0.68 \AA$. U., less than half as large. The radius of the iodide ion even, which is of the xenon type, is according to Bragg only $1.40 \AA$. U. Now one would expect on the basis of the Lewis-Langmuir theory that the outer shells of the sodium and fluoride ions are the same size and that of the iodide ion is much larger. In the alkali halides, however, the completed outer shells of the ions are probably considerably separated due to the presence of repulsive as well as attractive forces within the lattice. Evidentlv, Bragg's values for the atomic radii arbitrarily place responsibility for this free space separating the ions more largely upon the sodium ions, but on other metal ions as well. These, therefore, have abnormally large values. The reason why the values assigned to the negative ions are so small is that the same value must serve for cases in which the atoms of the negative elements are sharing electrons and are therefore very much closer together, there being no free space separating them.

As evidence that the assumption of constant atomic radius can be only approximately correct, the following may be cited. The distance between carbon atoms in diamond is $1.54 \AA$. U. In graphite, it is 1.45 $\AA$. U., ${ }^{13}$ a discrepancy of $0.09 \AA$. U. or $6 \%$. Again, in both metallic calcium and calcium oxide, the calcium atoms are arranged on a face-centered cubic lattice. The distance between nearest calcium atoms in the metal is $3.93 \AA$. U. ${ }^{14}$ The distance between the corresponding calcium atoms in the oxide, if these have a constant diameter might be expected to be

12 W. L. Bragg, Phil. Mag., [6] 40, 169 (1920).

"Debye and Scherrer, Physik. Z., 18, 291 (1917).

14 Hull, Phys. Rev., [2] 17, 42 (1921). 
much greater owing to the fact that oxide ions have been introduced into the lattice. Actually, it is considerably less, $3.32 \AA$. U. ${ }^{16}$ This difference amounts to $0.61 \AA$. U. The calcium atoms must be considered to have a radius at least $16 \%$ less in the oxide than in the metal. Since, in all probability, the metallic calcium atoms have 2 outer electrons which, in the oxide, have gone over to the oxygen atom, some such difference might have been expected. In spite of these drawbacks, however, Bragg's values for atomic radii do give rather good average agreement with the existing data, when they are applied to the distances between unlike atoms of compounds, if these atoms are adjacent in the crystal.

Evidence that the type of lattice must in certain cases be taken into account, is furnished by the results of Wilsey on the silver halides. ${ }^{3}$ Wilsey has found that silver chloride and bromide are simple cubic whereas the iodide crystallizes in the diamond type of lattice. The distances between nearest unlike atoms are, silver chloride $2.78 \AA$. U.; silver bromide $2.89 \AA$. U.; silver iodide $2.83 \AA$. U. The distance between silver and iodide ions is a little less than the distance between silver and bromide ions, although it is almost certain that the iodine atom is larger than the bromine atom. Clearly, it is due to the distinctive type of packing in the silver iodide crystal that the iodide ions appears to be the smaller.

The domain of an atom in a crystal in all probability depends on its state of combination, that is to say, on the number and the arrangement of electrons in its outer shell, as well as on the kind of atom concerned, and, in certain cases, on the arrangement of atoms in the crystal. Bragg assumes that everything but the kind of atom can be left out of account. The author, on the other hand, suggests that the particular kind of atom is unimportant, for atoms of the same rare-gas type, and that the contribution of an atom to the distance between it and another atom in a crystal depends upon the number and arrangement of electrons about its positive nucleus, upon the type of lattice, at least as this affects the latter, and, ordinarily, upon the charge carried by the atom. Apparently, the latter is sometimes also unimportant when the atoms are close-packed, as in the case of substances crystallizing in the diamond lattice (e.g., zinc sulfide and cuprous chloride).

Davey has recently analyzed the inter-atomic distances in the alkali halides from this point of view with success. ${ }^{16}$ This series and the series of substances crystallizing in the diamond lattice are the only ones sufficiently developed at the present time to allow of analysis.

\section{Summary}

As an alternative to W. L. Bragg's view that the distances between ${ }_{15}$ Davey and Hoffman, Phys. Rev., [2] 15, 333 (1920).

${ }^{16}$ Davey, ibid., [2] 18, 102 (1921). 
atoms in crystals can be expressed in terms of atomic radii characteristic only of the element concerned, it is suggested that the particular element is of secondary importance, for elements whose atoms are of the same rare-gas type, and that the contribution of an atom to the distance between it and another atom in a crystal depends upon the number and arrangement of electrons about its positive nucleus, upon the type of lattice, at least as this affects the latter, and, ordinarily, upon the magnitude of the charge carried by the atom.

The inter-atomic distances in substances crystallizing in the diamond type of lattice have been analyzed from this point of view.

PRINCETON, NEW JERSEY

[Contribution from the Gates Chemical Laboratory, California Institutet OF Technology, No. 11]

\section{THE CRYSTAL STRUCTURES OF COMPLEX CYANIDES OF POTASSIUM WITH ZINC, CADMIUM AND MERCURY}

By Roscoe G. DickINSON 1

Received February 13, 1922

\section{Introduction}

The complex cyanides $\mathrm{K}_{2} \mathrm{Zn}(\mathrm{CN})_{4}, \mathrm{~K}_{2} \mathrm{Cd}(\mathrm{CN})_{4}, \mathrm{~K}_{2} \mathrm{Hg}(\mathrm{CN})_{4}, \mathrm{Tl}_{2} \mathrm{Zn}(\mathrm{CN})_{4}$, and $\mathrm{T}_{2} \mathrm{Hg}(\mathrm{CN})_{4}$ are mentioned by Groth ${ }^{2}$ as probably crystallizing with cubic symmetry; but apparently only the first compound has been shown to be optically isotropic. In the work to be here described crystals of the first three of these compounds were prepared and found to be optically isotropic. Their structures were also determined by Xray methods. The relation of the new structures to those of some other crystals, as well as the magnitude of the interatomic distances, present some interesting features which will be pointed out in the latter part of this article.

\section{Materials and Methods of Experimentation}

Potassium zinc cyanide was prepared by adding a solution of potassium cyanide (imported "sodium free" potassium cyanide was used throughout) to zinc sulfate solution, washing the precipitate, and dissolving it in warm, conc. potassium cyanide solution, from which the product separated on cooling.

Potassium cadmium cyanide was prepared by adding ammonium hydroxide to cadmium nitrate solution, washing the precipitate, and dissolving it in potassium cyanide solution.

Potassium mercury cyanide was obtained by adding mercuric oxide to potassium cyanide solution, and recrystallizing the product.

In all cases the crystals used were obtained by slow evaporation of their aqueous

${ }^{2}$ National Research Fellow in Chemistry.

2 Groth, "Chemische Krystallographie," Engelmann, Leipzig, 1906. Vol. 1, p. $334, \mathrm{ff}$. 